Optical Society of America. S. P. Almeida, S. K. Case, and W. J. Dallas, "Multispectral size-averaged incoherent sp'atial filtering," Appl. Opt. 18, 4025-4029 (1979) . doi: 10.1364/ao.18.004025

\title{
Multispectral size-averaged incoherent spatial filtering
}

\author{
S. P. Almeida, S. K. Case, and W. J. Dallas
}

\begin{abstract}
A method of 2-D size-averaged incoherent recognition filtering is described. White light input illumination is used to multiplex chromatically a continuum of inputs differing in their spatial Fourier spectral magnifications. A two-grating dichromated-gelatin lateral-dispersion compensating device is inserted to correct chromatic dispersion introduced by a computer-generated hologram filter. The resulting registered recognition patterns are added to form a single effective filter size-averaged recognition peak. Using high efficiency compensating gratings and a bleached binary hologram allow the use of a relatively low-power light source.
\end{abstract}

\section{Introduction}

An optical spatial filter recognition system is sometimes too exact in its discrimination between inputs. ${ }^{1}$ Although structurally identical, two objects may be put into separate categories due to a small size difference. The system we work with is designed to classify diatoms (i.e., water algae). ${ }^{2}$ Within one species they are structurally uniform but can vary in size by about $15 \%$. The technique we describe compensates for this size variation in much the same way a multiply exposed average filter would. ${ }^{2,3}$ We achieve this effect not by multiple exposure but by multispectral illumination in the grating compensated ${ }^{4}$ incoherent spatial filtering system shown in Fig. 1. The system is chromatically compensated, not truly achromatized ${ }^{5}$ : the intensity PSF magnification is deliberately left as a function of wavelength.

The system functions as follows. The input transparency in Fig. 1 is illuminated by spatially incoherent white light. Immediately after the transparency we can visualize the wave field as consisting of a superposition of spatially identical images of differing colors. Each of these images will be processed by the optical system independently and slightly differently from the others. These differences are due to two effects-lateral (chromatic) dispersion and variation of the effective filter magnification.

When this work was done all authors were with Virginia Polytechnic Institute \& State University, Physics Department, Blacksburg, Virginia 24061; S. K. Case is now with University of Minnesota, Electrical Engineering Department, Minneapolis, Minnesota 55455; W. J. Dallas is now with Philips Forschungslaboratorium Hamburg, 2000Hamburg-54, Federal Republic of Germany; and S. P. Almeida is on leave at Universidade Federal de Pernambuco, Departmento de Fisica, 50000 Recife, Brazil.

Received 28 May 1977; revised manuscript received 11 July 1979.

0003-6935/79/234025-05\$00.50/0.

(C) 1979 Optical Society of America.
The lateral dispersion is due to the grating nature of the computer hologram and to the grating pair GR1 and GR2. By positioning these three elements properly along the optical axis, the total lateral dispersion of the system can be set to zero, i.e., the filtering outputs for each wavelength can be brought into register. $\mathrm{Re}$ maining then are the effective filter magnification differences.

In incoherent spatial filtering as in coherent filtering, the position of a spatial frequency in the filter plane, for a system of fixed geometry, depends on the wavelength of light used. Changing the wavelength changes the magnification of the object's Fourier spectrum. This wavelength effected mangification change may also be viewed as a change in the effective size of a filter in Fourier space-a given filter is effectively larger in blue light than it is in red light. If the filter is matched to one object magnification at one wavelength, a spatial filtering using a superposition of wavelengths is equivalent to a filtering using a superposition of effective filters of varying size, i.e., a size-averaged matched filtering.

In the following sections we first quantify the preceding qualitative introduction in a mathematical description of the optical system. We then describe experiments performed and show their results.

\section{Wavelength Dependence in Incoherent Spatial Filtering}

In this section we first look at the coherent PSF for a conventional spatial filtering system. Beginning with the coherent system, it is necessary to make evident an angular (chromatic) dispersion factor, which is important in realized partially coherent optical recognition systems. This angular factor disappears with an assumption of strict incoherence. We then make the transition to incoherent spatial filtering to describe the optical system's basic working principles. 


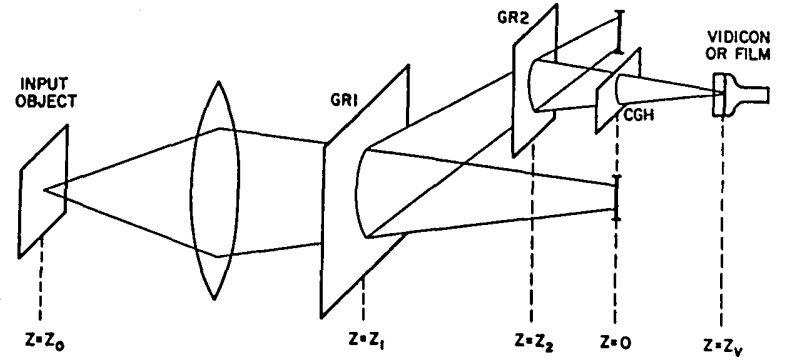

Fig. 1. Dispersion compensated size-averaging incoherent spatial filtering systems.

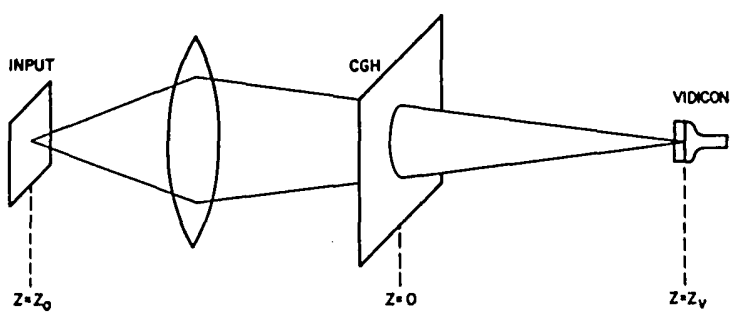

Fig. 2. Conventional incoherent spatial filtering system.

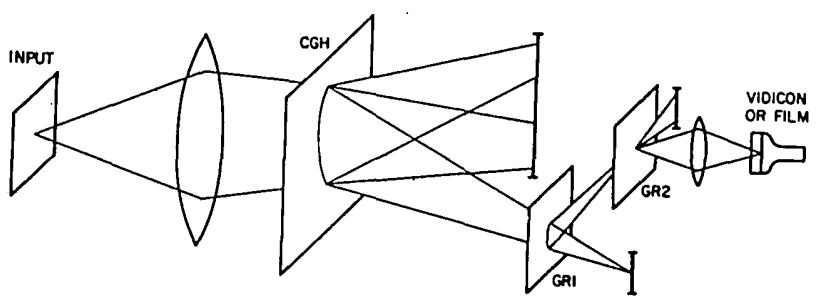

Fig. 3. Alternate filtering geometry.

Consider the conventional spatial filtering system illustrated in Fig. 2 (see Ref. 6). We specify the complex amplitude transmittance of the hologram to be $\tilde{v}(\mu, \nu)$, where

$$
\mu=-x / \lambda z_{v} \text { and } \nu=-y / \lambda z_{v} .
$$

The coherent PSF of the system is

$$
v_{1}(x, y ; \lambda)=\exp \left[i \pi\left(x^{2}+y^{2}\right) / \lambda z_{v}\right] v(x, y ; \lambda),
$$

where $v(x, y ; \lambda)$ is the inverse Fourier transform

$$
v(x, y ; \lambda)=\iint \tilde{v}\left(\mu^{\prime}, \nu^{\prime}\right) \exp \left[2 \pi i\left(\mu^{\prime} x+\nu^{\prime} y\right)\right] d x^{\prime} d y^{\prime},
$$

with $\left(\mu^{\prime}, \nu^{\prime}\right)$ related to $\left(x^{\prime}, y^{\prime}\right)$ by Eq. (1). Considering that, for some reference wavelength $\lambda_{0}$,

$$
v\left(x, y ; \lambda_{0}\right)=v(x, y),
$$

then

$$
v(x, y ; \lambda)=v(x / M, y / M) ; \quad M=\lambda / \lambda_{0},
$$

and the coherent PSF becomes

$$
v_{1}(x, y ; \lambda)=\exp \left[i \pi\left(x^{2}+y^{2}\right) / \lambda z_{v}\right] v(x / M, y / M) .
$$

Assuming $\tilde{v}(\mu, \nu)$ to be the coherent transfer function of a binary computer-generated hologram, the total PSF will be made up of a sum of images (the various hologram diffraction orders)

$$
v(x, y)=\sum_{n} u_{n}\left(x-n x_{0}, y\right),
$$

where we assume that the component images $u_{n}$ do not overlap. In this equation, $x_{0}$ is a constant inversely proportional to the resolution cell spacing in the hologram. Equation (6) thus becomes

$$
v_{1}(x, y ; \lambda)=\exp \left[i \pi\left(x^{2}+y^{2}\right) / \lambda z_{v}\right] \sum_{n} u_{n}\left(\frac{x-n M x_{0}}{M}, \frac{y}{M}\right) .
$$

This equation exhibits three types of wavelength dependence-magnification, lateral dispersion, and angular dispersion. The $M$ in the denominator of the $u_{n}$ arguments indicates a magnification change with wavelength; this magnification change we wish to keep. The term $n M x_{0}$ indicates a lateral dispersion, which means that, if we reconstruct in a nonzero diffraction order, the various PSF positions will vary with wavelength. This lateral dispersion is compensated by the grating pair GR1 and GR2 in Figs. 1 and 3.

The exponential coefficient in Eq. (8) shows an angular dispersion. For the system geometry shown in Fig. 1, this angular dispersion is unimportant. For truly incoherent light it would also be unimportant. However, in partially coherent light with alternate geometries (e.g., when an additional imaging stage is added), the dispersion becomes important in determining optical component parameters. The angular dispersion is described by the following relations. A normal to the average wave front will be at direction cosines

$$
\begin{aligned}
& \alpha=\cos \theta_{x}=\frac{\lambda}{2 \pi} \frac{\partial \Phi}{\partial x}=\frac{x}{z_{v}} \\
& \beta=\cos \theta_{y}=\frac{\lambda}{2 \pi} \frac{\partial \Phi}{\partial y}=\frac{y}{z_{v}}
\end{aligned}
$$

to the $x$ and $y$ axes, respectively, where $\Phi=\pi\left(x^{2}+\right.$ $\left.y^{2}\right) / \lambda z_{v}$. The lateral dispersion thus gives rise to an angular dispersion through the position dependence expressed in Eq. (9). The intensity PSF will be

$$
I_{\text {tot }}(x, y ; \lambda)=\sum_{n}\left|u_{n}\left(\frac{x-n M x_{0}}{M}, \frac{y}{M}\right)\right|^{2}
$$

before lateral dispersion compensation. We consider, henceforth, only the first-order diffraction term

$$
I(x, y ; \lambda)=\left|u_{1}\left(\frac{x}{M}-x_{0}, \frac{y}{M}\right)\right|^{2},
$$

as the intensity PSF. We may disregard the other diffraction orders as long as the sums of corresponding dimensions of the input field and first-order PSF are less than the spacing between diffraction orders. Choice of the first hologram diffraction order is not mandatory. For inexpensive implementation of a large input field system, a higher diffraction order ${ }^{7}$ is chosen $(n \neq 1)$. By decomposing the total PSF differently from that in Eq. (10), it may even be shown that $n$ need 
not be integer, i.e., the recognition may be performed between the integer diffraction orders of the hologram in "fractional" orders. ${ }^{8}$

An important well-known property of incoherent recognition systems is that they are insensitive to lateral displacement of the filter. Upon Fourier transforming Eq. (11), one obtains an autocorrelation as the effective filter transmittance for the first diffraction order. The autocorrelation of any function $f$ is, however, invariant with displacement of $f$ :

$$
\int f\left(x^{\prime}\right) f^{*}\left(x^{\prime}-x\right) d x^{\prime}=\iint f\left(x^{\prime}-x_{0}\right) f^{*}\left(x^{\prime}-x_{0}-x\right) d x^{\prime} .
$$

This shift insensitivity is important in the filter geometry of Fig. 1, where the optical axis (as defined by a normally exiting ray in the input plane) is laterally displaced for the different colors.

\section{Double Diffraction Compensation of Lateral Dispersion}

The pair of gratings GR1 and GR2 compose a lateral dispersion compensating device. The important parameters are evident from a short calculation. We consider gratings of possibly different spatial frequency and possibly rotated with respect to one another. The grating spatial frequencies we note by $\left(\mu_{1}, \nu_{1}\right)$ and $\left(\mu_{2}, \nu_{2}\right)$ for GR1 and GR2, respectively. One design parameter is that the lateral beam dimension at the hologram is smaller than the correlation function of interest. Fulfilling this condition allows the GR1 and GR2 diffraction orders to be separated and undesired orders blocked. As illustrated in Fig. 1, only the plus first order from GR1 and minus first order from GR2 are used. Let

$$
u\left(x, y, z^{-} ; \lambda\right)=u(x, y)
$$

be the complex amplitude incident on GR1. We note its Fourier transform (angular spectrum) by $\tilde{u}(\mu, \nu)$. By applying the usual propagation equations, we arrive at the angular spectrum exiting from the second grating as being

$$
\begin{aligned}
\tilde{u}\left(\mu, \nu, z_{2}^{+} ; \lambda\right)= & \tilde{u}(\mu, \nu) \exp \left\{2 \pi i\left[\left(\mu_{2}-\mu_{1}\right) x+\left(\nu_{2}-\nu_{1}\right) y\right]\right\} \\
& \cdot \exp \left(\frac{2 \pi i \Delta z}{\lambda}\left\{1-\lambda^{2}\left[\left(\mu-\mu_{1}\right)^{2}+\left(\nu-\nu_{1}\right)^{2}\right]^{1 / 2}\right\}\right) .
\end{aligned}
$$

Expanding the square root to second order in $(\mu, \nu)$ yields

$$
\begin{aligned}
\sqrt{ }= & {\left[1-\lambda^{2}\left(\mu_{1}^{2}+\nu_{1}^{2}\right)\right]^{1 / 2}+\frac{\lambda^{2}\left(\mu_{1} \mu+\nu_{1} \nu\right)}{\left[1-\lambda^{2}\left(\mu_{1}^{2}+\nu_{1}^{2}\right)\right]^{1 / 2}} } \\
& -\frac{\lambda^{2}\left(\mu^{2}+\nu^{2}\right)}{2\left[1-\lambda^{2}\left(\mu_{1}^{2}+\nu_{1}^{2}\right)^{1 / 2}\right.} \frac{\lambda^{4}\left(\mu_{1} \mu+\nu_{1} \nu\right)^{2}}{8\left[1-\lambda^{2}\left(\mu_{1}^{2}+\nu_{1}^{2}\right)\right]^{3 / 2}} .
\end{aligned}
$$

The first term is an unimportant constant. The last term expresses the fact that this is a somewhat astigmatic imaging system, a fact that will limit the useful space-bandwidth product of the system. For now we drop the first term and ignore the last. Inverse transforming gives the complex amplitude exiting the second grating as

$$
u\left(x-x_{a}, y-y_{a}, z_{1}+z_{a}\right) \cdot \exp 2 \pi i\left[\left(\mu_{2}-\mu_{1}\right) x+\left(\nu_{2}-\nu_{1}\right) y\right],
$$

$$
\left.\begin{array}{l}
x_{a}=\frac{-\lambda \mu_{1} \Delta z}{\left[1-\lambda^{2}\left(\mu_{1}^{2}+\nu_{1}^{2}\right)\right]^{1 / 2}} ; \quad y_{a}=\frac{-\lambda \nu_{1} \Delta z}{\left[1-\lambda^{2}\left(\mu_{1}^{2}+\nu_{1}^{2}\right)\right]^{1 / 2}} ; \\
z_{a}=\frac{\Delta z}{\left[1-\lambda^{2}\left(\mu_{1}^{2}+\nu_{1}^{2}\right)\right]^{1 / 2}}
\end{array}\right\}
$$

The parameter $z_{a}$ expresses the slight change in focus due to the added propagation path. This change in focus is

$$
\Delta z_{f}=z_{a}-\Delta z
$$

The important features of Eq. (16) are the lateral dispersion and the angular dispersion. Lateral dispersion comes from the functional relationships of $\left(x_{a}, y_{a}\right)$ to $\lambda$. The angular dispersion is expressed by the exponential in expression (16a). Analogously to Eq. (9),

$$
\alpha=\lambda\left(\mu_{2}-\mu_{1}\right), \quad \beta=\lambda\left(\nu_{2}-\nu_{1}\right) .
$$

Particularizing to

$$
\mu_{2}=\mu_{1}, \quad \nu_{2}=\nu_{1}=0,
$$

the angular dispersion disappears, and we have

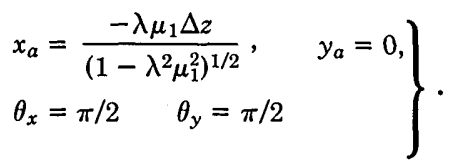

Inserting this result into Eq. (11) we have

$$
I(x, y)=\left|u_{1}\left(\frac{x-M x_{0}-x_{a}}{M}, \frac{y}{M}\right)\right|^{2}
$$

to compensate lateral dispersion:

$$
\frac{\lambda}{\lambda_{0}} x_{0}+x_{a}=0, \quad x_{0}=\frac{\lambda_{0} \mu_{1} \Delta z}{\left(1-\lambda^{2} \mu_{1}^{2}\right)^{1 / 2}} .
$$

If the lateral dimension of the resolution cell in the hologram is $\Delta x$, the corresponding spatial frequency is

$$
\mu_{c}=1 / \Delta x, \quad x_{0}=\mu_{c} \lambda z_{v},
$$

giving a necessary grating separation of

$$
\Delta z=z_{v} \frac{\lambda}{\lambda_{0}} \frac{\mu_{c}}{\mu_{1}}\left(1-\lambda^{2} \mu_{1}^{2}\right)^{1 / 2} .
$$

\section{Size-Averaged Correlation}

After dispersion compensation, the interesting portion of the intensity PSF from the filter will be

$$
I_{F}(x, y ; \lambda)=\left|u_{1}(x / M, y / M)\right|^{2} .
$$

An object in the input plane whose intensity transmittance is $I_{0}(x, y)$ would, when illuminated by quasimonochromatic incoherent light, give rise to an output cross-correlation

$$
\begin{aligned}
C(x, y ; \lambda)= & \left(I_{0} \otimes I_{F}\right)\left(x-x_{0}, y ; \lambda\right) \\
= & \iint_{-\infty}^{\infty} I_{0}\left[x^{\prime}-\left(x-x_{0}\right), y^{\prime}-y\right] \\
& \times I_{F}\left(x^{\prime} / M, y^{\prime} / M\right) d x^{\prime} d y^{\prime} .
\end{aligned}
$$

If a spectral distribution $S(\lambda)$ propagates to the output plane, the total output is 


$$
C_{t}(x, y)=\int_{0}^{\infty} S(\lambda) C(x, y ; \lambda) d \lambda
$$

Interchanging the order of integration gives

$$
\left.\begin{array}{l}
C_{t}(x, y)=\left(I_{0} \otimes I_{\mathrm{av}}\right)\left(x-x_{0}, y\right) \\
I_{\mathrm{av}}(x, y)=\int_{0}^{\infty} S(\lambda) I_{F}\left(\frac{\lambda_{0}}{\lambda} x^{\prime}, \frac{\lambda_{0}}{\lambda} y^{\prime}\right) d \lambda
\end{array}\right\}
$$

The resulting output is the correlation between the input and a size-averaged PSF $I_{\text {ave }}$ The weighting function of this average is simply the spectral distribution $S(\lambda)$. This spectral distribution can be modified by a color filter inserted anywhere in the system.

\section{Alternate Geometries}

Figure 3 shows an alternate arrangement of the system components, which differs in no essential properties from the system shown in Fig. 1. The experimental results shown in the following section were obtained with this alternate geometry-there is no deeper reason,

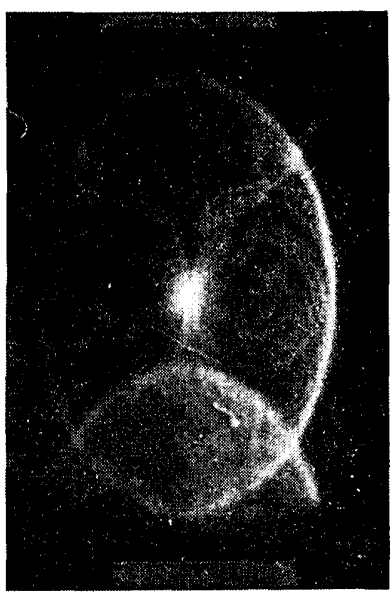

(a)

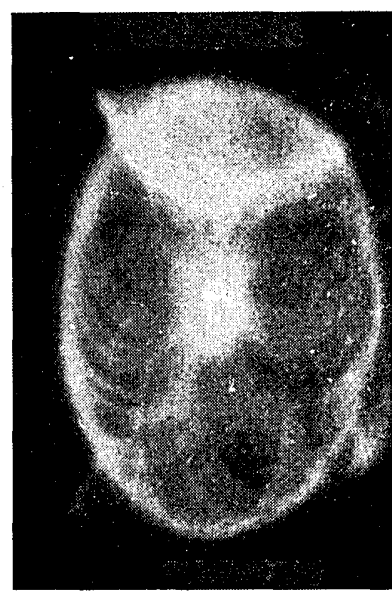

(c)

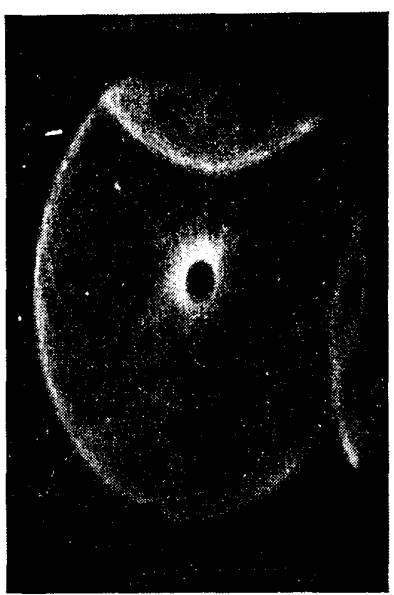

(b)

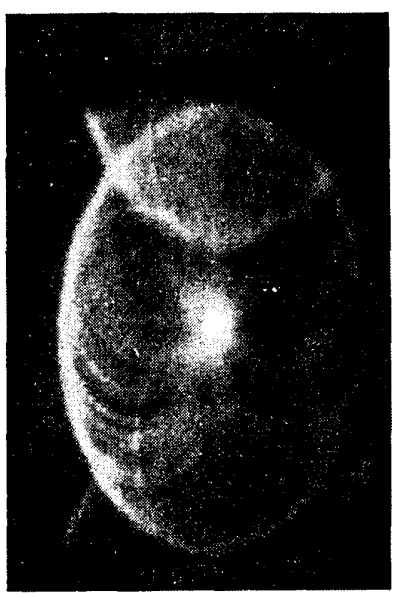

(d)
Fig. 4. Correlation peak for 2:1 aspect-ratio ellipse: (a) sizematched; (b) $20 \%$ size mismatch; (c) size averaged with broad weighting function; and (d) size averaged with narrow weighting function.

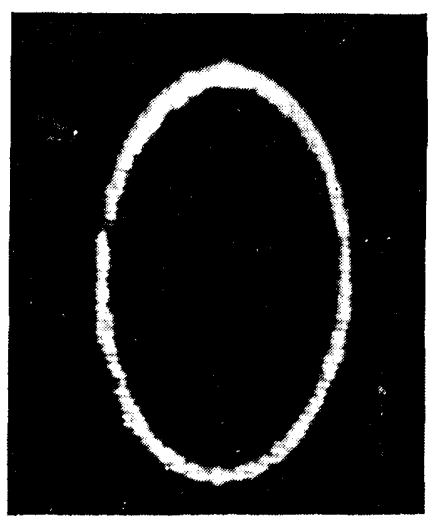

Fig. 5. Input transparency for filtering results shown in Fig. 4.

the system was simply so arranged when it came time to take the pictures. Yet another possible arrangement is to use a single relatively high spatial-frequency compensating grating at a small distance from the image plane. The disadvantage of this arrangement when compared with the two compensating grating configurations of Figs. 1 and 2 is that, although the single grating compensates the lateral dispersion, a rather large angular dispersion is introduced. This angular dispersion makes imaging with subsequent stages very difficult. Another modification of the system described is to use two gratings in contact ${ }^{9}$ to replace the grating GR2 of Fig. 1. Relatively rotating the gratings gives an effective moiré grating allowing compensation of both lateral and angular dispersions.

\section{Experiment}

The experimental results shown in Fig. 4, using the input transparency shown in Fig. 5, were obtained with the system configuration of Fig. 3 .

The original object is a computer synthesized ellipse. A binary computer-generated Fourier hologram plot was generated of this ellipse and replicated on an offset press. A $4 \times 4$ replicated hologram plot was photoreduced onto $35-\mathrm{mm}$ film, which was then bleached to increase diffraction efficiency. The input transparency is a $1.5-\mathrm{mm}$ square photograph of the reconstructed image from this hologram.

The input transparency was illuminated by imaging the active area of a $2-W$ zirconium arc lamp (Oriel Optics) onto the transparency via a microscope objective. The first (transforming) lens was a $180-\mathrm{mm}$ focal length camera objective, the second (imaging) lens was a camera objective mounted on the camera (with bellows extension).

The gratings GR1 and GR2 were holographically generated $800 \mathrm{lp} / \mathrm{mm}$ in dichromated gelatin. These were nearly $100 \%$ diffraction efficient for blue light. It is the remarkable efficiency of these gratings plus the high efficiency of the bleached binary computer-generated hologram which made possible the relatively low light source power requirement. The system was so arranged that the filter was matched to the input for green light. Figure 4(a) shows the correlation between 
the system PSF and the input for this matched wavelength. A narrowband interference filter was inserted into the system near the computed hologram to select this wavelength.

Figure 4(b) shows the results of using another wavelength (red) corresponding to a $20 \%$ size mismatch between the input and filter. The hollow elliptical shape of this peak is the cross-correlation between two ellipses of significantly differing size. In Fig. 4(c) no chromatic filter was inserted in the system. This result in white light shows a broad but quite distinct size-averaged recognition peak. In Fig. 4(d) a broadband green dichroic filter (Edmund Scientific) was inserted near the computer hologram and effects a size averaging over a $10 \%$ range of sizes.

The authors gratefully acknowledge the National Science Foundation's support of this work through grant NSF-ENV 72-03581-J03.

\section{Addendum}

During this paper's dormant period an interesting article on using chromatic multiplexing for 1-D recognition has appeared. ${ }^{10}$

\section{References}

1. D. Casasent and D. Psaltis, Appl. Opt. 15, 1795 (1976).

2. S. P. Almeida and J. K.-T. Eu, Appl. Opt. 15, 510 (1976).

3. J. C. Viénot, J. Duvernoy, G. Tribillon, J.-L. Tribillon, Appl. Opt. 12, 950 (1973).

4. D. J. DeBitetto, Appl. Phys. Lett. 9, 417 (1966).

5. R. H. Katyl, Appl. Opt. 11, 1255 (1972).

6. J. D. Armitage and A. W. Lohmann, Appl. Opt. 4, 461 (1965).

7. H. Bartelt, W. J. Dallas, and A. W. Lohmann, Opt. Commun. 20, 50 (1977).

8. H. Becker and W. J. Dallas, Opt. Commun. 15, 50 (1975).

9. A. W. Lohmann and O. Bryngdahl, Appl. Opt. 6, 1934 (1967).

10. J.-P. Goedgebuer and R. Gazeu, Opt. Commun. 27, 53 (1978).

\section{Variable-shape solar energy concentrator}

In a proposed low-cost 3-D tracking solar concentrator, a stretched aluminized membrane is supported on a lightweight hoop and enclosed in a lightweight transparent plastic dome that protects it from the elements. Since the membrane concentrator would be considerably lighter than conventional rigid concentrators, it could be driven by a relatively inexpensive low-power tracking mechanism.

As shown in Fig. 4, the aluminized (polymeric-film) membrane is supported by a hoop fastened to a lightweight backing dish. The dish and membrane enclose a sealed volume, and a vacuum can be drawn in this volume to change the membrane shape. The aluminized reflecting surface can be adjusted to a parabolic contour, or to other shapes, by differential pressure loading. The loading is varied either by fabricating a membrane with a nonuniform radialthickness distribution and applying uniform pressure, or a nonuniform pressure distribution can be applied by adding distributed weights to a membrane of uniform thickness.

To create a parabolic shape, the loading should be uniform on a unit area of a plane perpendicular to the axis of the parabola. Local curvature corrections can be made by placing electrets or magnetic materials near selected locations on the convex undersurface of the film and applying electric or magnetic fields to pull the membrane into the desired shape.

Although the unit has yet to be constructed and tested, its relatively simple design and potential for low-cost fabrication and operation indicate that the concept warrants further development.

This work was done by Charles G. Miller of Caltech and Jens H. Pohl of California Polytechnic State University of San Luis Obispo for Jet Propulsion Laboratory. Refer to NPO-13736.

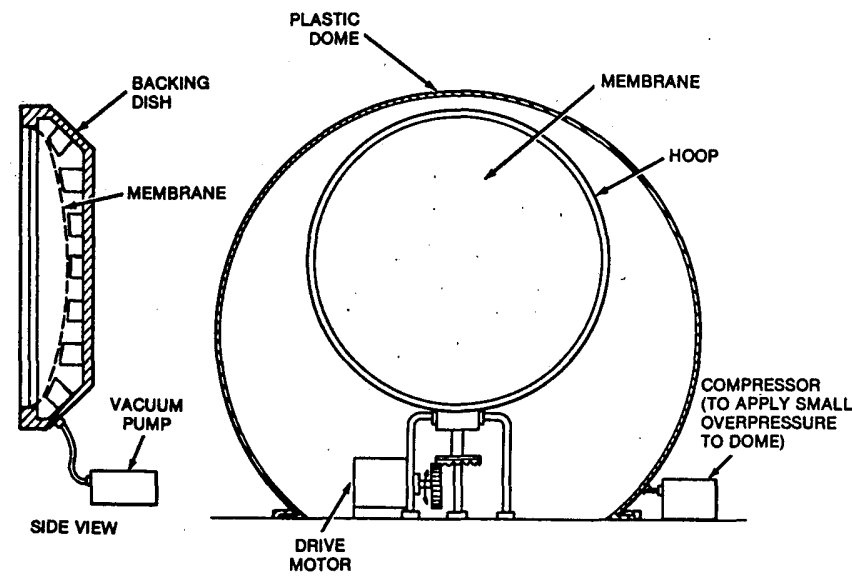

Fig. 4. This lightweight solar concentrator would be fabricated from a flexible polymeric-film membrane. The membrane shape is controlled by differential pressure loading. Fine adjustments to the shape can be made by mounting electrets or magnets on the membrane and applying electric or magnetic fields. 